(6)

OPEN ACCESS

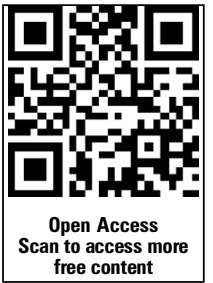

- Additional material is published online only. To view please visit the journal online (http://dx.doi.org/10.1136/ heartjnl-2015-307798)

${ }^{1}$ Department of Medicine, Levanger Hospital, NordTrøndelag Health Trust, Levanger, Norway

${ }^{2}$ Department of Heart Disease, Haukeland University Hospital, Bergen, Norway ${ }^{3}$ Department of Clinical Science, Faculty of Medicine and Dentistry, University of Bergen, Bergen, Norway ${ }^{4} \mathrm{MI}$ Lab, Department of Circulation and Medical Imaging, Norwegian University of Science and Technology, Trondheim, Norway

\section{Correspondence to} Dr Havard Dalen, Levanger Hospital, Nord-Trøndelag Health Trust, Levanger 7600, Norway;

havard.dalen@ntnu.no

Received 10 March 2015 Revised 24 August 2015 Accepted 13 September 2015 Published Online First 5 October 2015

\section{SLinked}

- http://dx.doi.org/10.1136/ heartinl-2015-308236 - http://dx.doi.org/10.1136/ heartjnl-2015-308802

\section{CrossMark}

To cite: Gundersen GH, Norekval TM, Haug HH, et al. Heart 2016;102: 29-34.

\title{
Adding point of care ultrasound to assess volume status in heart failure patients in a nurse-led outpatient clinic. A randomised study
}

\author{
Guri Holmen Gundersen, ${ }^{1}$ Tone M Norekval, ${ }^{2,3}$ Hilde Haugberg Haug, ${ }^{1}$ Kyrre Skjetne, ${ }^{1}$ \\ Jens Olaf Kleinau, ${ }^{1}$ Torbjorn Graven, ${ }^{1}$ Havard Dalen ${ }^{1,4}$
}

\section{ABSTRACT}

Objectives Medical history, physical examination and laboratory testing are not optimal for the assessment of volume status in heart failure (HF) patients. We aimed to study the clinical influence of focused ultrasound of the pleural cavities and inferior vena cava (IVC) performed by specialised nurses to assess volume status in HF patients at an outpatient clinic.

Methods HF outpatients were prospectively included and underwent laboratory testing, history recording and clinical examination by two nurses with and without an ultrasound examination of the pleural cavities and IVC using a pocket-size imaging device, in random order. Each nurse worked in a team with a cardiologist. The influence of the different diagnostic tests on diuretic dosing was assessed descriptively and in linear regression analyses.

Results Sixty-two patients were included and 119 examinations were performed. Mean \pm SD age was 74 \pm 12 years, $E F$ was $34 \pm 14 \%$, and $N$-terminal pro-brain natriuretic peptide (NT-proBNP) value was 3761 $\pm 3072 \mathrm{ng} / \mathrm{L}$. Dosing of diuretics differed between the teams in 31 out of 119 consultations. Weight change and volume status assessed clinically with and without ultrasound predicted dose adjustment of diuretics at follow-up ( $p<0.05)$. Change of oedema, NT-proBNP, creatinine, and symptoms did not $(p \geq 0.10)$. In adjusted analyses, only volume status based on ultrasound predicted dose adjustments of diuretics at first visit and follow-up (all ultrasound $p \leq 0.01$, all other $p \geq 0.2$ ). Conclusions Ultrasound examinations of the pleural cavities and IVC by nurses may improve diagnostics and patient care in HF patients at an outpatient clinic, but more studies are needed to determine whether these examinations have an impact on clinical outcomes. Trial registration number NCT01794715.

\section{INTRODUCTION}

In spite of optimal medical therapy, heart failure (HF) may progress with unpredictable episodes of worsening. Fluid retention (hypervolaemia) is a major cause of worsening HF. ${ }^{1-3}$ Haemodynamic disturbances leading to acute decompensated HF may start weeks before the actual onset of typical $\mathrm{HF}^{1}{ }^{1}$ In the early stages of decompensation effective and more aggressive therapy could potentially prevent deterioration and hospitalisation. ${ }^{4}$ Estimation of volume status is crucial because achieving euvolaemia in HF patients is an important goal of therapy. ${ }^{1}$ Diuretics are superior for achieving and maintaining euvolaemia but frequently there is a very narrow window of optimal volume status. ${ }^{5}{ }^{6}$ Hyper- and hypovolaemia may lead to decompensated HF and organ failure caused by inadequate perfusion. ${ }^{2}$

Traditionally, the cornerstone of the patient's volume status assessment in the outpatient HF clinic is the evaluation of symptoms, monitoring of weight and peripheral oedema, and blood tests including creatinine and natriuretic peptides, but these are neither sensitive nor specific with respect to detecting volume status. ${ }^{3-10}$ Ultrasound (US) is a sensitive and specific tool to estimate volume status, by assessment of the inferior vena cava (IVC) and the pleural cavities, and may have a crucial influence. ${ }^{10-14}$ The development of high quality, low cost pocket-size imaging devices (PSID) has promoted diagnostic US by non-experts. ${ }^{15-17}$ It is not known if the PSIDs could be used as a tool for monitoring volume status and guiding treatment in HF patients. We have recently shown that specialised nurses in an HF clinic can perform point of care US for quantification of pleural effusion and the dimension and inspiratory collapsibility of the IVC, with a high agreement with cardiologists using high-end equipment. ${ }^{16}$ Specialised nurses have a growing role in diagnostics and follow-up of outpatient HF patients, ${ }^{18}$ and adding point of care US diagnostics to the nurses' consultations may improve care. Thus, we aimed to study the clinical influence of focused US of the pleural cavities and IVC performed by specialised nurses, to assess volume status in patients with HF in a nurse-led outpatient clinic.

\section{PATIENTS AND METHODS}

\section{Study population and design}

Patients allocated to the outpatient HF clinic at the non-university Levanger Hospital, Norway, 15 April to 21 June 2013 were eligible for inclusion. Written informed consent was obtained. The study was registered at ClinicalTrials.gov (ID: NCT01794715) and approved by the Regional Committee for Medical and Health Research Ethics (REK 2013/257), and conducted according to the Second Declaration of Helsinki. Follow-up data are shown in online supplementary results.

Figure 1 shows the patient flow and the crossover design of the study, and the basis for the statistical analyses. At each study visit the patients were examined by two separate specialised nurses both 
with and without point of care US. The sequence of the examinations with and without US, as well as which personnel performed US, was in each case randomised by draw. Each of the two nurses were blinded to the information from the other nurse. For each visit the nurse worked in a team with a cardiologist (one of four) in a random order. After the patients had been examined by one of the nurses at each study visit, they were examined by the second nurse, either with or without US opposite to the first nurse. Further details on the training of the nurses have been recently published. ${ }^{16}$

The specialised nurses recorded the medical history and performed a clinical examination with respect to physical signs of fluid retention or depletion at every visit to the HF clinic. During each patient visit, one of the nurses carried out point of care US of the pleural cavities and IVC to assess volume status as an adjunct to the clinical examination, and the other nurse completed the medical history recording and the clinical examination without point of care US. The nurses discussed their findings with respect to adjusting therapy with a cardiologist in a random way (figure 1). The therapeutic decisions made by the 'non-US' team were applied for the study purpose only and the patients were treated according to the decision of the team which included US in the consultation.

Only the team (nurse and cardiologist) who included the US examination had access to the US findings. If the patient later visited the HF clinic for follow-up, the US examination was performed by the nurse only and no echocardiography was performed by a cardiologist. At follow-up visits, both teams had access to previous US findings.

Blood samples were drawn from the participants at baseline and follow-up. The blood samples were analysed at the hospital's IEC 17025 accredited laboratory for assessment of haemoglobin, sodium, potassium, international normalised ratio (INR), uric acid, creatinine, estimated glomerular filtration rate (eGFR), and N-terminal pro-brain natriuretic peptide (NT-proBNP).

\section{History recording and clinical examination to assess volume status}

The history recording included assessment of the patients' symptoms according to New York Heart Association (NYHA) classification by both nurses. The patients were assigned NYHA class I-IV where I refers to no symptoms and IV refers to symptoms at rest. The clinical examination by the two specialised nurses was performed in a random order in separate rooms.

The clinical examination included: ECG, measurement of blood pressure and heart rate, weight control, and assessment of peripheral oedema. Weight change since hospital discharge or a previous visit in the HF clinic was classified as weight loss or weight gain if it was reduced or increased by $>1.5 \mathrm{~kg}$ and as no change in between. Peripheral oedema was graded as no oedema, ankle oedema, leg oedema or oedema above the knee. No cardiac auscultation or further clinical assessment was performed by the nurses.

\section{Point of care US examinations by the nurses to assess volume status}

The US examination was performed using the Vscan system (GE Ultrasound, Horten, Norway). With the patient in the supine position the dimensions of the IVC were measured distally to the inlet of the hepatic vein in the sagittal plane. The complete respiratory cycle was recorded, including sniff.

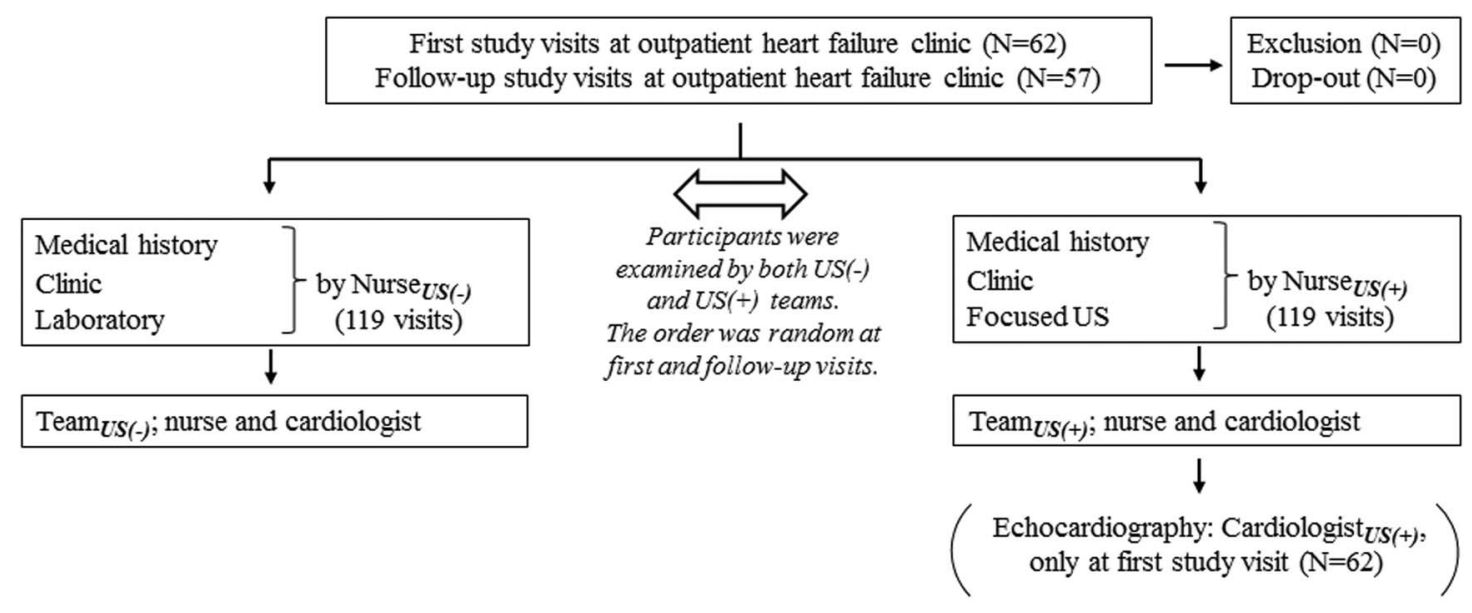

\begin{tabular}{|c|c|c|c|}
\hline \multicolumn{4}{|c|}{ Basis for statistical analyses } \\
\hline$\downarrow$ & I & $\downarrow$ & 7 \\
\hline $\begin{array}{c}\text { Medical history } \\
\text { (NYHA-class) } \\
(\mathrm{N}=119)\end{array}$ & $\begin{array}{c}\text { Clinical examination } \\
\text { (oedema) } \\
(\mathrm{N}=119)\end{array}$ & $\begin{array}{c}\text { Volume status } \\
\text { (medical history }+ \text { clinic }+ \\
\text { laboratory } \pm \text { US })(\mathrm{N}=119)\end{array}$ & $\begin{array}{c}\text { Diuretic dosing } \\
\text { (medical history }+ \text { clinic }+ \\
\text { laboratory } \pm \text { US })(N=119)\end{array}$ \\
\hline $\begin{array}{c}\text { Nurse }_{U S(-)} \\
\text { vs Nurse } \\
U S(+)\end{array}$ & $\begin{array}{c}\text { Nurse }_{U S(-)} \\
\text { vs }_{\text {Nurse }} \text { US (+) }_{(+)}\end{array}$ & $\begin{array}{c}\text { Nurse }_{U S(-)} \\
\text { vS }_{\text {Nurse }} \text { US (+) }_{(+)}\end{array}$ & $\begin{array}{c}\text { Team }_{U S(-)} \\
\text { vs } \operatorname{Team}_{U S(+)}\end{array}$ \\
\hline
\end{tabular}

Figure 1 Flow chart of the study. All participants underwent full cross-over with examinations with and without ultrasound (US) in a random order at every study visit. The sequence was randomised by draw both at first and follow-up study visits. Echocardiography for validation was only performed at first visit $(\mathrm{N}=62)$. Both the teams with and without access to US made therapeutic decisions based on all available information (usual care \pm US examination). N, number of visits; NYHA, New York Heart Association; US(-), without access to ultrasound; US(+), with access to ultrasound. 
Trailing-to-leading edge (ie, the inner diameter) was used. The collapsibility index of the IVC (IVC $\left.{ }_{C I}\right)$ was calculated as the difference between end-expiratory and end-inspiratory dimension divided by the end-expiratory dimension. The IVC was divided into five categories by adding the score for the maximal dimension and collapsibility. For end-expiratory dimension score 1, 2 and 3 refer to $I_{V C} C_{\max }<1.7 \mathrm{~cm}, 1.7-2.1 \mathrm{~cm}$ and $>2.1 \mathrm{~cm}$, respectively, and for the $\mathrm{IVC}_{\mathrm{CI}}$ score 1,2 and 3 refer to $\geq 50 \%$, $35-50 \%$ and $<35 \%$, respectively. ${ }^{12} 19$

With the patient in a sitting position both hemithoraces were examined with the US transducer placed intercostally from the paravertebral to the axillary lines. The presence of pleural effusion was diagnosed by the appearance of an echo-free space between the diaphragm and the lung and the amount was measured between the diaphragm and the lung surface in the middle between the transducer and the mediastinum.

Additional data are provided in the online supplementary methods.

\section{Influence on treatment}

The nurses classified the patients' volume status based on the complete examination (with or without US) as hypovolaemic, euvolaemic or hypervolaemic. Any decisions regarding diuretic adjustment were made in cooperation with the cardiologist on the basis of all available data from both the laboratory tests, and clinical and US examinations. Dosing of diuretics was classified as: -1 , reduced; 0 , unchanged; or +1 , increased diuretic dose.

\section{Validation by high-end echocardiography}

Reference echocardiography was performed by one of four experienced cardiologists using a Vivid 7 scanner (GE Ultrasound) immediately after the US examination by the nurses and included assessment of the IVC and pleural cavities as described above. The high feasibility and high reliability of the US examinations by the nurses is comprehensively described in a recent publication. ${ }^{16}$

\section{Statistical analysis}

Normal distributed data are presented as mean $\pm S D$ if not described elsewhere. The comparison of different clinical and US indices for assessing HF (ie, symptoms, oedema, dimension or grading of pleural effusion, assessment of volume status) between the nurses, nurses and cardiologists, or between the two teams was analysed by Spearman's or Pearson's correlation coefficient, as well as kappa statistics. Data are presented as $\mathrm{r}$ (95\% CI) with the 95\% CI computed using bootstrapping. Predictors of diuretic therapy were tested in uni- and multivariate linear regression analyses with dose adjustments (reduced $(-1)$, unchanged (0) or increased $(+1)$ intensity) used as dependent variables and the different predictors of clinical interest to test were included as covariates. In sensitivity analyses using logistic regression models, predictors for increased or decreased diuretic dosing were tested. In addition, the clinical influence of EF and whether the patient was seen by a cardiologist was tested. A two-sided $\mathrm{p}$ value $<0.05$ was considered statistically significant. All the statistical analyses were performed using SPSS for Windows (V.21, SPSS, Inc).

\section{RESULTS}

\section{Study population}

The clinical characteristics of the 62 study participants are shown in table 1 . Mean \pm SD age was $74 \pm 12$ years, and $\mathrm{EF}$ and NT-proBNP were $34 \pm 14 \%$ and $3761 \pm 3072 \mathrm{ng} / \mathrm{L}$, respectively. The main reasons for not using angiotensin receptor blockers
Table 1 Clinical characteristics of the 62 study participants

\begin{tabular}{ll}
\hline & Mean \pm SD (range) \\
\hline Age, years & $74 \pm 12(35-91)$ \\
Women, $\mathrm{n}(\%)^{*}$ & $30(48)^{*}$ \\
Body mass index $\left(\mathrm{kg} / \mathrm{m}^{2}\right)$ & $28.3 \pm 5.5(18.6-45.8)$ \\
Systolic blood pressure (mm Hg) & $121 \pm 23(80-171)$ \\
Diastolic blood pressure (mm Hg) & $71 \pm 14(50-107)$ \\
Sinus rhythm, $\mathrm{n}(\%)^{*}$ & $25(40)^{*}$ \\
Heart rate, bpm & $79 \pm 21(51-140)$ \\
NT-proBNP, ng/L & $3761 \pm 3072(90-9999)$ \\
eGFR, mL/min/1.73 m ${ }^{2}(C o c k c r o f t-G a u l t)$ & $34 \pm 15(12-85)$ \\
NYHA functional class, median (25th and 75 th centile) & $11(\mathrm{I}$ and III)* \\
Cause of heart failure; ischaemic, $\mathrm{n}(\%)^{*}$ & $31(50)^{*}$ \\
Cause of heart failure; mainly diastolic, $\mathrm{n}(\%)^{*}$ & $15(24)^{*}$ \\
Using diuretics, $\mathrm{n}(\%)^{*}$ & $56(90)^{*}$ \\
Using $\beta$-blockers, $\mathrm{n}(\%)^{*}$ & $49(79)^{*}$ \\
Using ACEl or ARB, $\mathrm{n}(\%)^{*}$ & $37(60)^{*}$ \\
EF (\%) & $33.7 \pm 13.6(7-64)$ \\
LV end-diastolic dimension, mm & $56 \pm 11(22-80)$ \\
LV end-diastolic volume, mL & $136 \pm 76(35-398)$ \\
Mitral early inflow, cm/s & $83 \pm 25(39-144)$ \\
Mitral early inflow deceleration time, ms & $176 \pm 47(82-263)$ \\
Any pleural effusion, $\mathrm{n}(\%)^{*}$ & $26(42)^{*}$ \\
\hline
\end{tabular}

${ }^{*}$ Data not presented as mean \pm SD (range), and thus specified. $\mathrm{ACEl}$, angiotensin-converting enzyme inhibitor; ARB; angiotensin receptor blockers, eGFR; estimated glomerular filtration rate, $n$; number; NT-proBNP; N-terminal pro-brain natriuretic peptide; NYHA; New York Heart Association.

(ARBs) and angiotensin-converting enzyme inhibitors (ACEIs) at study entry was renal failure in $27 \%$, hypotension in $36 \%$, and diastolic HF in $9 \%$, while in the rest of the patients such medications were started later. The distribution of pleural effusion was not associated with EF $(p=0.10)$. The high morbidity and mortality of the participants is shown in the online supplementary results.

\section{Reliability of medical history, clinical signs, physical findings, and US}

Table 2 shows the relatively poor agreement between medical history, clinical signs and physical findings assessed by the two specialised nurses. The nurses agreed in the assessment of volume status in 75 out of 119 paired consultations. The two teams (nurses and cardiologists) agreed in somewhat more (89 out of 119) paired consultations regarding diuretic dosing.

Table 2 Agreement of different indices relevant for heart failure assessment in 119 crossover consultations by the two nurses

\begin{tabular}{lll}
\hline & Agreed, $\mathbf{n}(\%)$ & Agreement $(\mathbf{r} / \mathbf{k})$ \\
\hline NYHA class & $97(82)$ & 0.67 \\
Peripheral oedema & $88(74)$ & 0.59 \\
Volume status $^{\text {Diuretic treatment }}{ }^{*}$ & $75(63)$ & 0.38 \\
\hline
\end{tabular}

The table shows the fair to substantial agreement between the two nurses of different indices relevant for heart failure assessment (all $p<0.001$ ). Volume status assessed by clinical findings, laboratory tests and with or without focused ultrasound examination. Agreed describes the number (\%) with exact match, while the agreement by Spearman correlation statistics takes weighted difference into account. *Agreement is between the two teams (nurse and cardiologist with and without access to ultrasound, respectively).

NYHA, New York Heart Association; r, correlation; $\kappa$, kappa statistics. 
Figure 2 shows the high correlations of the nurses' measurements of the dimension of the IVC and quantification of the pleural effusion with the reference measurements. During the first visit pleural effusion was detected in 36 pleural cavities in 23 patients by the nurses and in 39 pleural cavities in 26 patients by reference echocardiography performed by the cardiologists.

\section{Predictors of diuretic therapy}

The online supplementary table shows the characteristics and clinical findings of 17 follow-up visits where diuretic treatment differed between the teams (with and without US for the assessment of volume status). It is shown that an increase of pleural effusion revealed with PSID resulted in an increase of diuretic therapy, and correspondingly a reduction of pleural effusion resulted in a decrease of diuretic therapy.

Table 3 shows predictors of diuretic therapy by the first study visit and by follow-up. At the first visit, volume status assessed as cumulative information of clinical and laboratory tests, as well as assessment of IVC or the pleural cavities by US, predicted diuretic dosing, but as standalone indices neither clinical nor laboratory tests predicted diuretic dosing. By the follow-up visits, weight change and all ultrasonographic indices were also significant predictors of dose adjustment of diuretic therapy, whereas change in the distribution of oedema, NT-proBNP, creatinine or change of NYHA class did not predict diuretic dose adjustment (all $\mathrm{p} \geq 0.10$ ). In analyses adjusted for all described variables, only volume status based on US predicted change of diuretic dosing when this variable was entered at the first step and the non-US variables were entered stepwise thereafter (all US $p \leq 0.01$, all other $p \geq 0.2$ ). Whether or not the cardiologist performed echocardiography as reference imaging (only at first visit) did not significantly influence the result $(\mathrm{p}=0.12)$.

The semi-quantitative classification of pleural effusion and IVC correlated well with volume status (both $r=0.67$, $\mathrm{p}<0.001)$. At first study visit both the findings of any pleural effusion or a dilated IVC with reduced collapsibility with semiquantitative score $\geq 5$ were associated with increased mortality with 2 year follow-up ( $\mathrm{p}=0.07$ and $\mathrm{p}<0.001$, respectively) (see online supplementary results and figure).

During mean follow-up of 38 days only three (5\%) patients were admitted due to any HF related events. At 1 year HF readmissions were $25.8 \%$. Additional data are presented in the online supplementary results.

\section{DISCUSSION}

In this study of patients in an outpatient HF clinic, specialised nurses were able to perform and interpret point of care US examination of the IVC and the pleural cavities to assess volume status with excellent quality, in contrast to the poor interobserver agreement of medical history, clinical signs and physical examination. In regression analyses, the estimated volume status based on US predicted dose adjustments of diuretics significantly better than any other tool used to assess patients' volume status and guide therapy.

Patients with HF are encumbered with substantial morbidity and this was also the case in this study population. Pleural effusion is closely related to congestion and decompensation among HF patients and may be difficult to detect both by clinical examination and chest X-ray. ${ }^{14}$ However, pleural effusion is easily assessable by US and at first visit pleural effusion was present in $42 \%$ of the patients. Detection of newly developed pleural effusion in HF patients may indicate that the patients need intensified treatment. As shown by the basic characteristics the study population is quite similar to populations in other HF clinics, and it is reasonable to believe that our frequent finding of pleural effusion may be present in most HF populations. ${ }^{14} 20$

The present study shows poor to moderate correlation between the nurses regarding medical history and physical signs. Although the clinical assessment of peripheral oedema and classification of NYHA class are commonly used in the HF clinic, neither of them has been proven to be sufficiently objective, reliable or sensitive. ${ }^{21} 22$ Symptoms and signs of fluid retention may be minimal and unspecific, and even attenuated by a low level of daily activity. ${ }^{14}$ In addition, comorbidity and venous insufficiency may be misleading.

However, in the present study neither weight change, oedema, blood tests nor NYHA class appear optimal when it comes to monitoring of volume status. Monitoring the data may perform better than discrete values. However, the routine use of US may improve the assessment of volume status. The agreement between the teams (nurse and cardiologist) regarding volume status was numerically somewhat better compared to the agreement between the nurses, respectively.
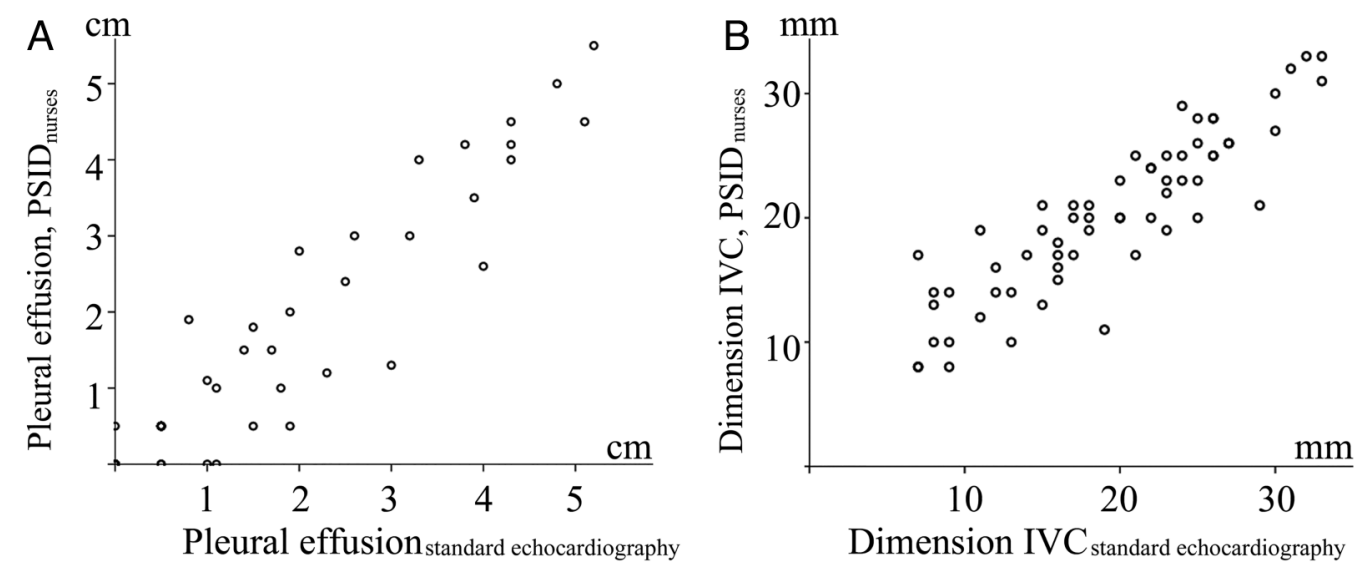

Figure 2 Correlation of ultrasound indices to assess volume status by nurses with reference. The figure shows correlation of: (A) quantification of pleural effusion measured as the dimension of fluid between the diaphragm and the lung surface with patients in sitting position; and (B) end-expiratory dimension of the inferior vena cava (IVC) by pocket-size imaging device (PSID) examinations performed by nurses plotted against similar measurements by reference echocardiography. In $(A)$ no effusion measured by both the nurse and reference is shown as the dot at $0 ; 0$ and the dot at $0.5 ; 0.5$ refers to effusion in the costodiaphragmatic recess only measured by both users. 
Table 3 Predictors of diuretic therapy at first visit and follow-up study visits

\begin{tabular}{|c|c|c|c|c|c|c|}
\hline \multirow[b]{2}{*}{ Covariates } & \multicolumn{3}{|c|}{ First study visit } & \multicolumn{3}{|c|}{ Follow-up study visits } \\
\hline & $\boldsymbol{\beta}$ & $R^{2}$ & p Value & $\boldsymbol{\beta}$ & $\mathrm{R}^{2}$ & p Value \\
\hline Volume status included US & 0.576 & 37.5 & $<0.001$ & 0.575 & 39.1 & $<0.001$ \\
\hline IVC category & 0.290 & 20.7 & $<0.001$ & 0.139 & 6.2 & $<0.001$ \\
\hline IVC dimension & 0.263 & 11.5 & 0.01 & 0.302 & 18.6 & $<0.001$ \\
\hline Pleural effusion & 0.109 & 9.0 & 0.02 & 0.885 & 13.0 & $<0.01$ \\
\hline Volume status without US & 0.284 & 7.5 & 0.03 & 0.349 & 14.0 & $<0.01$ \\
\hline Weight & 0.002 & 0.4 & 0.63 & 5.43 & 12.0 & $<0.01$ \\
\hline Oedema & 0.107 & 2.0 & 0.27 & 0.126 & 2.7 & 0.22 \\
\hline NYHA class & 0.187 & 2.6 & 0.21 & 0.168 & 3.9 & 0.14 \\
\hline Creatinine & -0.001 & 0.5 & 0.60 & -0.430 & 2.0 & 0.29 \\
\hline NT-proBNP & 0.000 & 0.5 & 0.10 & 0.018 & 1.6 & 0.35 \\
\hline
\end{tabular}

In a recent study, we showed the high correlations of quantitative measurements of pleural effusion and the dimension of IVC between specialised nurses and cardiologists. ${ }^{16}$ This result is in line with findings of other groups with more or less inexperienced users after dedicated training. ${ }^{15} 1723$

The robustness observed by the pocket-sized imaging of the pleural cavities and the IVC may be related to the fact that these measurements are not too difficult to obtain with high accuracy. This shows that the training of the nurses was sufficient and in line with recent recommendations, ${ }^{24-26}$ and that the applied US assessment seems attractive for the monitoring of patients with $\mathrm{HF}^{13} 1427$

To our knowledge, no other study has shown the clinical influence of routinely including focused US of the pleural cavities and the IVC, performed by specialised nurses, to assess volume status and guide treatment in an outpatient HF clinic. However, whether the implementation of focused US imaging of the pleural cavities and the IVC to assess volume status for guidance of therapy will exert an influence on mortality and morbidity remains to be shown. In this study, given the high morbidity of the HF patients, only three patients (5\%) were hospitalised during the study period (mean 38 days). This appears to be a low number compared to published data where readmission rates at 30-60 days were $10-50 \% .^{4} 27$ The hospitalisation rate in the present study is in line with the results from studies where implantable devices for haemodynamic monitoring have been used to guide treatment. ${ }^{28}$

Previous studies have shown that US assessment of the IVC and pleural cavities is able to predict right atrial filling pressures, predict outcome, and may very well be suited for the monitoring of HF patients. ${ }^{10} 121420$ Pleural effusion is frequently present in asymptomatic worsening of HF, with minimal appearance of other HF-related signs. ${ }^{10}$ Implementing the described US examination may have an important influence on therapeutic decision making and potentially also the patient's outcome. Natriuretic peptides have a high specificity for diagnosing HF, provide important prognostic information, and are able to guide therapy in HF patients. However, their performance may be inferior to US examinations for individual monitoring for follow-up of HF patients. ${ }^{27}$ Natriuretic peptide guided therapy may be most useful to optimise the doses of $\beta$-blockers and ACEIs. $^{29} 30$
The high prevalence of pleural effusion among HF patients, the high feasibility and accuracy of US examination by nurses, and the high availability and low cost of the PSID may result in a high level of clinical gain by implementing focused US for the assessment of volume status in HF patients. Restricting such diagnostic examinations only to physicians may limit the clinical gain.

\section{Limitations}

This study was a single centre study. The major limitations are the lack of a gold standard for assessing volume status and the lack of clinical endpoints. The US findings of significant pleural

\section{Key messages}

\section{What is already known on this subject?}

Fluid retention (hypervolaemia) is a major cause of morbidity and readmissions and may start weeks before the actual onset of typical heart failure (HF) symptoms. Evaluation of symptoms, monitoring of weight and peripheral oedema, blood tests and chest X-ray are not sensitive to detecting early changes in volume status.

\section{What might this study add?}

We have shown the clinical influence of routinely including focused ultrasound of the pleural cavities and the inferior vena cava, performed by specialised nurses, to assess volume status and guide treatment in an outpatient HF clinic. In this study nurses were able to perform and interpret point of care ultrasound to assess volume status with excellent quality, allowing for improvement in available information for the caregiving physician.

\section{How might this impact on clinical practice?} Implementation of point of care ultrasound to assess volume status may improve diagnostics, and thus follow-up and prognosis among heart failure patients. However, whether this will have an influence on mortality and morbidity remains to be shown. 
effusion or a very narrow IVC with a very high CI may significantly alter decisions regarding diuretic dosing and this may have influenced the result. Further studies investigating the influence of this methodology on endpoints such as readmission rates and mortality are warranted.

\section{CONCLUSION AND IMPLICATIONS}

Focused US examinations of the pleural cavities and IVC among HF patients in an outpatient clinic performed by nurses significantly predicted dose adjustments of diuretics compared to standard care. Acknowledging the lack of a gold standard for assessing volume status, we conclude that implementing point of care US appears attractive for optimising the management of HF patients beyond standard clinical care, but further studies are needed to determine whether point of care US improves clinical outcomes.

Contributors All the authors fulfil all the listed criteria below: (1) conception and design or analysis and interpretation of data, or both; (2) drafting of the manuscript or revising it critically for important intellectual content; (3) final approval of the manuscript submitted.

Funding The Nord-Trøndelag Health Trust, Norway, funded this study.

Competing interests HD holds position at the Medical Imaging Laboratory, NTNU, a Centre of Research-based Innovation that is funded by the Research Council of Norway and industry. One of the industry partners is GE Ultrasound. The Centre had a total budget of 124 million NOK for the 8-year period 2007-2014, and the contribution from GE Vingmed Ultrasound to this budget is approximately 7 million NOK (6\%).

Patient consent Obtained.

Ethics approval Regional Committee for Medical and Health Research Ethics.

Provenance and peer review Not commissioned; externally peer reviewed.

Data sharing statement Additional unpublished data regarding patients' QoL are available, though not analysed.

Open Access This is an Open Access article distributed in accordance with the Creative Commons Attribution Non Commercial (CC BY-NC 4.0) license, which permits others to distribute, remix, adapt, build upon this work non-commercially, and license their derivative works on different terms, provided the original work is properly cited and the use is non-commercial. See: http://creativecommons.org/ licenses/by-nc/4.0

\section{REFERENCES}

1 Pang PS. Pathophysiology of volume overload in acute heart failure syndromes. Congest Heart Fail 2010;16:51-6.

2 Albert NM. Fluid management strategies in heart failure. Critic Care Nurse 2012:32:20-32.

3 von Lueder TG, Krum H. Current modalities for invasive and non-invasive monitoring of volume status in heart failure. Heart 2012;98:967-73.

4 Desai AS, Stevenson LW. Rehospitalization for heart failure: predict or prevent? Circulation 2012:126:501-6.

5 Faris RF, Flather $\mathrm{M}$, Purcell $\mathrm{H}$, et al. Diuretic treatment, chronic heart failure. Cochrane Library. 2012.

6 McMurray JJ, Adamopoulos S, Anker SD, et al. ESC Guidelines for the diagnosis and treatment of acute and chronic heart failure 2012: The Task Force for the Diagnosis and Treatment of Acute and Chronic Heart Failure 2012 of the European Society of Cardiology. Eur Heart J 2012;33:1787-847.

7 Fonseca C, Morais H, Mota T, et al. The diagnosis of heart failure in primary care: value of symptoms and signs. Eur J Heart Fail 2004;6:795-800.
8 Mueller C, Frana B, Rodriguez D, et al. Emergency diagnosis of congestive heart failure: impact of signs and symptoms. Can J Cardiol 2005;21:921-4.

9 Rohde LE, Beck-da-Silva L, Goldraich L, et al. Reliability and prognostic value of traditional signs and symptoms in outpatients with congestive heart failure. Can J Cardiol 2004;20:697-702.

10 Kataoka H. Utility of thoracic sonography for follow-up examination of chronic heart failure patients with previous decompensation. Clin Cardiol 2007;30:336-41.

11 Blehar DJ, Dickman E, Gaspari R. Identification of congestive heart failure via respiratory variation of inferior vena cava diameter. Am J Emerg Med 2009:27:71-5.

12 Brennan JM, Blair JE, Goonewardena S, et al. Reappraisal of the use of inferior vena cava for estimating right atrial pressure. I Am Soc Echocardiogr 2007:20:857-61.

13 Pellicori P, Carubelli V, Zhang J, et al. IVC diameter in patients with chronic heart failure: relationships and prognostic significance. JACC Cardiovasc Imaging 2013;6:16-28.

14 Kataoka H. Ultrasound pleural effusion sign as a useful marker for identifying heart failure worsening in established heart failure patients during follow-up. Congest Heart Fail 2012;18:272-7.

15 Andersen GN, Viset A, Mjolstad OC, et al. Feasibility and accuracy of point-of-care pocket-size ultrasonography performed by medical students. BMC Med EduC 2014:14:156.

16 Dalen $\mathrm{H}$, Gundersen GH, Skjetne $\mathrm{K}$, et al. Feasibility and reliability of pocket-size ultrasound examinations of the pleural cavities and vena cava inferior performed by nurses in an outpatient heart failure clinic. Eur J Cardiovasc Nurs 2015;14:286-93.

17 Mjolstad OC, Andersen GN, Dalen H, et al. Feasibility and reliability of point-of-care pocket-size echocardiography performed by medical residents. Eur Heart J Cardiovasc Imaging 2013;14:1195-202.

18 Sauer J, Rabelo ER, Castro RA, et al. Nurses' performance in classifying heart failure patients based on physical exam: comparison with cardiologist's physical exam and levels of N-terminal pro-B-type natriuretic peptide. J Clin Nurs 2010;19:3381-9.

19 Rudski LG, Lai WW, Afilalo J, et al. Guidelines for the echocardiographic assessment of the right heart in adults. J Am Soc Echocardiogr 2010;23:685-713.

20 Goonewardena SN, Gemignani A, Ronan A, et al. Comparison of hand-carried ultrasound assessment of the inferior vena cava and $\mathrm{N}$-terminal pro-brain natriuretic peptide for predicting readmission after hospitalization for acute decompensated heart failure. JACC Cardiovasc Imaging 2008;1:595-601.

21 Brodovicz KG, McNaughton K, Uemura N, et al. Reliability and feasibility of methods to quantitatively assess peripheral edema. Clin Med Res 2009;7:21-31.

22 Raphael C, Briscoe C, Davies J, et al. Limitations of the New York Heart Association functional classification system and self-reported walking distances in chronic heart failure. Heart 2007:93:476-82.

23 Panoulas VF, Daigeler AL, Malaweera AS, et al. Pocket-size hand-held cardiac ultrasound as an adjunct to clinical examination in the hands of medical students and junior doctors. Eur Heart J Cardiovasc Imaging 2013;14:323-30.

24 Neskovic AN, Edvardsen T, Galderisi M, et al. Focus cardiac ultrasound: the European Association of Cardiovascular Imaging viewpoint. Eur Heart I Cardiovasc Imaging 2014;15:956-60.

25 Sicari R, Galderisi M, Voigt JU, et al. The use of pocket-size imaging devices: a position statement of the European Association of Echocardiography. Eur J Echocardiogr 2011;12:85-7.

26 Platz E, Solomon SD. Point-of-care echocardiography in the accountable care organization era. Circulation Cardiovasc Imaging 2012;5:676-82.

27 Carbone F, Bovio M, Rosa GM, et al. Inferior vena cava parameters predict re-admission in ischemic heart failure. Eur J Clin Invest 2014;44:341-9.

28 Abraham WT, Adamson PB, Bourge RC, et al. Wireless pulmonary artery haemodynamic monitoring in chronic heart failure: a randomised controlled trial. Lancet 2011;377:658-66.

29 Lainchbury JG, Troughton RW, Strangman KM, et al. N-terminal pro-B-type natriuretic peptide-guided treatment for chronic heart failure: results from the BATTLESCARRED (NT-proBNP-Assisted Treatment To Lessen Serial Cardiac Readmissions and Death) trial. J Am Coll Cardiol 2009;55:53-60.

30 Felker GM, Hasselblad V, Hernandez AF, et al. Biomarker-guided therapy in chronic heart failure: a meta-analysis of randomized controlled trials. Am Heart J 2009;158:422-30. 